omentum was then transposed to cover the mediastinum and the Dacron graft through the extended upper abdominal approach. The skin and subcutaneous tissues were resutured, with the defect in the sternum left. After the operation, the patient required mechanical ventilation for respiratory failure for 8 days. After endotracheal extubation, she recovered well. She has been doing well for 28 months after the operation.

\section{Comment}

Pseudoaneurysm of the brachiocephalic artery has been reported from a variety of etiologies. ${ }^{1}$ Traumatic injuries are the most frequently reported cause of this pseudoaneurysm. Blunt or stabbing injury ${ }^{2,3}$ and medically introduced trauma, ${ }^{4}$ such as catheterization, intravenous cannulation, and stenting, have occasionally been reported as causes. Infectious cause has infrequently been seen after cardiac surgery. ${ }^{5}$ Cannulation sutures or artificial grafts have become the foci of mediastinitis and have caused mycotic pseudoaneurysm of adjacent arteries.

Our patient showed mediastinitis and pseudoaneurysm of the brachiocephalic artery long after the surgical treatment for invasive thymoma. Because of the extent of the mediastinitis and the pus formation in the implanted graft, the pseudoaneurysm of the brachiocephalic artery appeared to be of infectious origin. The chronic mediastinitis was associated with previous median sternotomy and postoperative irradiation, although more than 5 years had passed since the resection of invasive thymoma. Adjuvant irradiation would have caused poor vascularization in the postoperative mediastinum, with consequent failure of protection against transsternal infection. Such a mycotic pseudoaneurysm of brachiocephalic artery after combined surgery and irradiation has seldom been reported in the literature. Extensive procedures-including removal of infective foci, necrotic sternum, and artificial graft; excision of the pseudoaneurysm; surgical irrigation; and omentum transposition-appear to have been successful in treating this extended mediastinal infection.

\section{References}

1. Kieffer E, Chieche L, Koskas F, Bahnini A. Aneurysms of the innominate artery: surgical treatment of 27 patients. J Vasc Surg. 2001;34: 222-8.

2. de Jose Maria B, Gomar C, Mestres C, Sorribes V, Moral V, Sala X. Pseudoaneurysm of the brachiocephalic artery caused by blunt chest trauma. J Thorac Cardiovasc Surg. 1995;110:863-5.

3. Reddi AA, Munasur MM, Naidoo RR, Steer DD. Traumatic innominate artery aneurysm 26 years after stab injury. Ann Thorac Surg. 2005;79: 1034-6.

4. Shield CF, Richardson JD, Buckley CJ, Hagood CO Jr. Pseudoaneurysm of the brachiocephalic arteries: a complication of percutaneous internal jugular vein catheterization. Surgery. 1975;78:190-4.

5. Katsumata T, Moorjani N, Vaccari G, Westaby S. Mediastinal false aneurysm after thoracic aortic surgery. Ann Thorac Surg. 2000;70: $547-52$.

\title{
Pulmonary segmental venous infarction after living- donor lobar lung transplantation
}

\author{
Cliff K. Choong, FRACS, Ding Z. Hu, MD, and Charles B. Huddleston, MD, St Louis, Mo
}

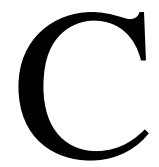

omplete obstruction of one or more of the major pulmonary veins after lung transplantation is a devastating complication. It results in hemorrhagic infarction of the affected lung within 4 to 6 hours, leading to irreversible lung damage. Reported treatments have included resection of the affected lobe and retransplantation. ${ }^{1,2}$

\footnotetext{
From the Division of Cardiothoracic Surgery, Department of Surgery, Washington University School of Medicine, St Louis, Mo.

Received for publication Jan 29, 2005; accepted for publication March 14, 2005.

Address for reprints: Charles B. Huddleston, MD, Professor of Surgery, No. 1 Children's Place, Suite 5S 50, Children's Hospital, St Louis, MO 63110 (E-mail: huddlestonc@msnotes.wustl.edu).

J Thorac Cardiovasc Surg 2005;130:919-21

$0022-5223 / \$ 30.00$

Copyright $\odot 2005$ by The American Association for Thoracic Surgery

doi:10.1016/j.jtcvs.2005.03.045
}

We present a case of pulmonary venous infarction after transplantation that was treated conservatively.

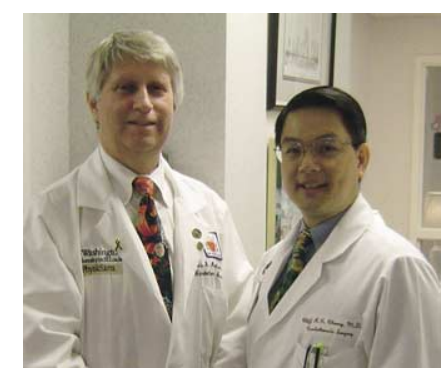

Huddleston and Choong (left to right)

\section{Clinical Summary}

A 16-year-old girl with a history of cystic fibrosis, multiple pulmonary infections, insulin-dependent diabetes, malnutrition, and anemia underwent a bilateral living-donor lobar lung transplantation for the treatment of end-stage lung disease secondary to cystic fibrosis. Before the operation, she had a forced expiratory volume of $20 \%$ predicted and was severely limited by her poor respiratory function. She had to use a wheelchair and required continuous supplementary oxygen. The surgery was performed with cardiopulmonary bypass support and proceeded uneventfully. The donor right and left lower lobes were implanted into the recipient right and left hemithoraces, respectively. Her chest radiograph after the operation was unremarkable, apart from mild bilateral diffuse infiltrates. On postoperative day 1 , her chest radiograph revealed a confluent opacity involving the apical segment of the right lower lobe 

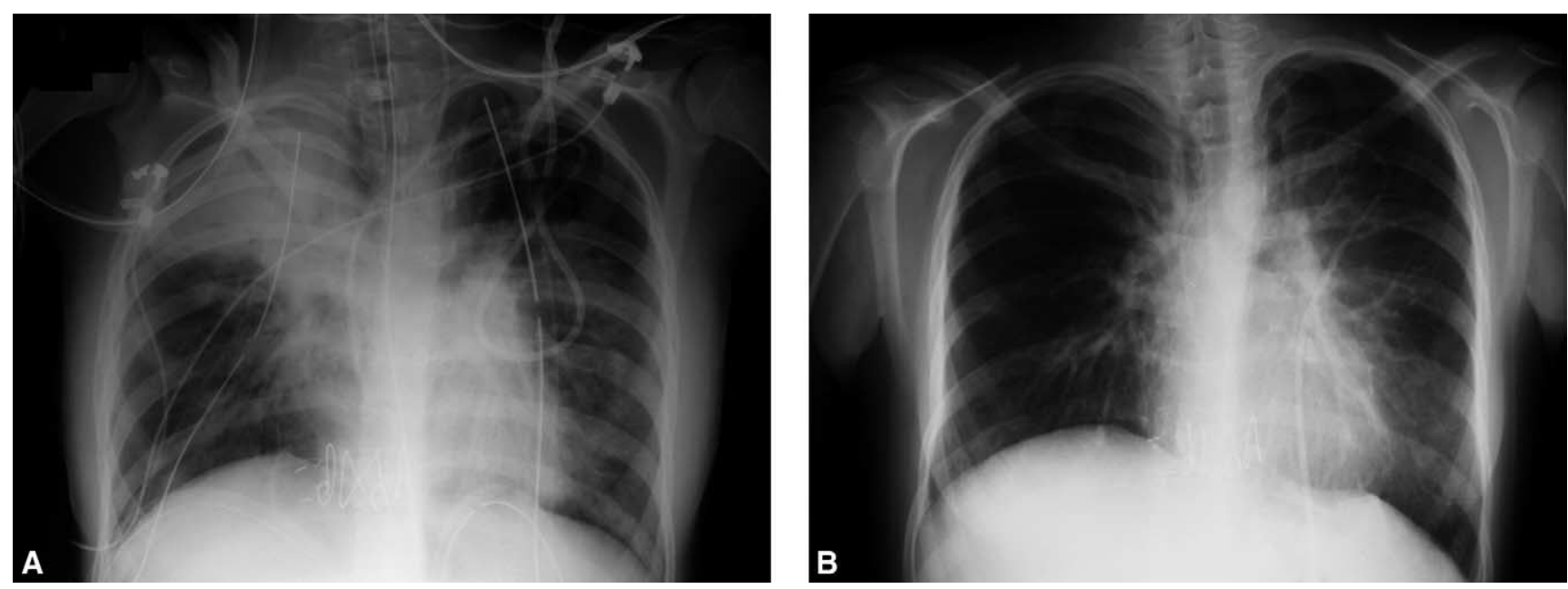

Figure 1. A, Chest radiograph showing confluent opacity involving apical segment of donor right lower lobe. B, Chest radiograph 6 weeks after lung transplantation showing complete resolution of right apical segmental opacity. Right hemidiaphragm is elevated because of volume loss secondary to resultant fibrosis of apical segment.

(Figure 1, A). Examination with a flexible bronchoscope revealed bloody secretions in the right apical segmental airway. Pulmonary perfusion scintigraphy showed that the right lung received $56 \%$ of the total pulmonary perfusion, but there was a marked decreased perfusion in the upper portion of the right lung. Angiography revealed an obstruction involving the vein draining the apical segment of the right lower lobe (Figure 2, A). A diagnosis of segmental pulmonary venous infarction was made. The obstruction was probably related to technical issues at the time of donor harvest or implantation. The patient was treated conservatively because the infarction had involved only one segment of the donor lungs. Her postoperative course was also complicated by pulmonary edema, pulmonary infection, and renal failure, and she required postoperative mechanical ventilatory support for 7 days. She was discharged on day 28 without any requirement for supplementary oxygen. The right apical segmental opacity on the chest radiograph gradually resolved during a 6-week period (Figure 1,B). Pulmonary
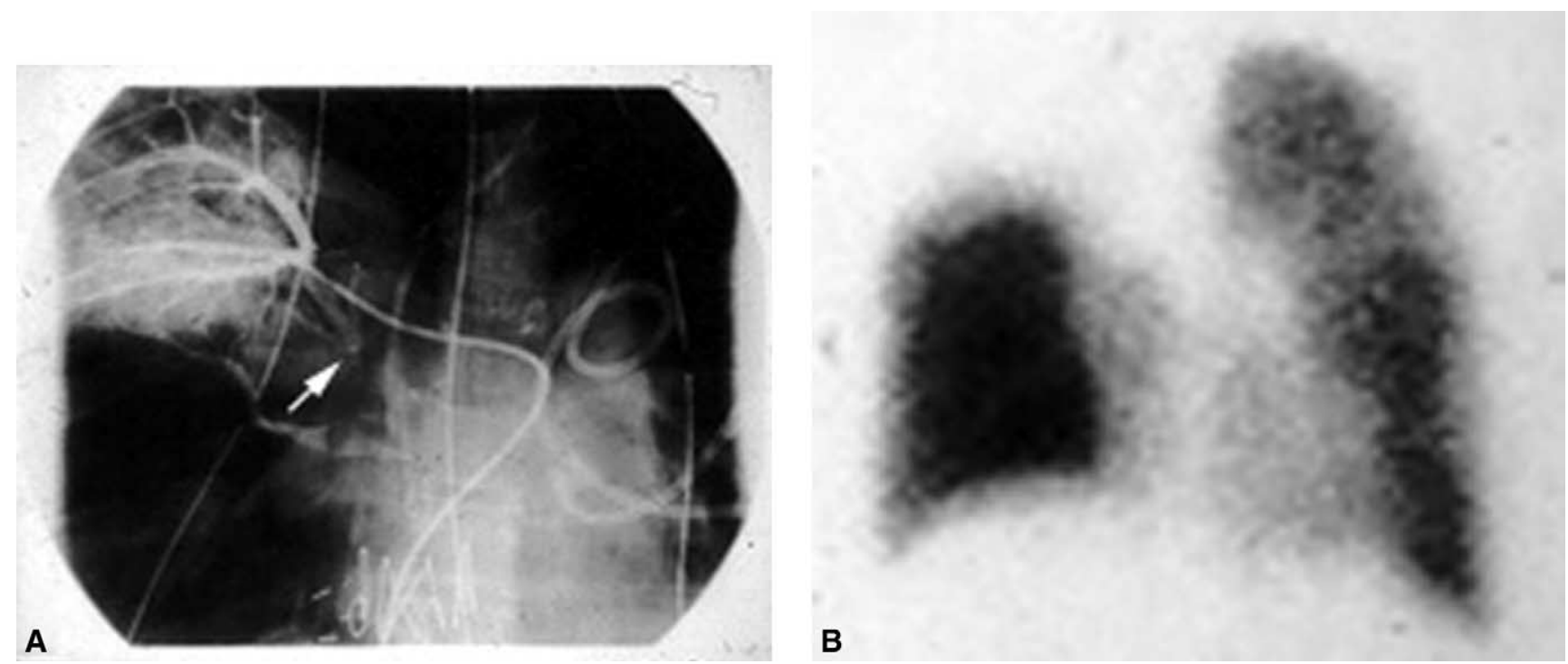

Figure 2. A, Selective pulmonary angiography showing abrupt occlusion (arrow) of vein draining right apical segment. B, Pulmonary perfusion scintigraphy 3 months after transplantation showing persistent markedly decreased perfusion in upper portion of right lung. 
perfusion scintigraphy 3 months after the transplantation showed persistent, marked decreased perfusion in the upper portion of the right lung (Figure 2,B). She has remained well through a follow-up period of 2 years, with excellent functional health.

\section{Discussion}

Pulmonary venous infarction after lung transplantation is a rare but life-threatening condition. The causes in lung transplantation have been obstruction of the pulmonary vein by thrombosis formed secondary to a narrowing of the venous anastomosis in the setting of a lack of bronchial arterial blood supply. ${ }^{1,2}$ The clinical and radiologic features may be mistaken for reperfusion injury, consolidation of the lung, or myocardial dysfunction. The diagnosis is often delayed, resulting in the onset of hemorrhagic infarction and irreversible lung damage. Early diagnosis and prompt treatment are therefore essential. This, however, seldom occurs, because it is difficult to diagnose pulmonary venous infarction by conventional roentgenologic means. The findings on chest radiography are nonspecific and variable and include pulmonary consolidation, atelectasis, increased pulmonary vascular markings, and increased hilar size. ${ }^{3}$ Perfusion scan is useful in demonstrating a marked decreased perfusion in the affected area. ${ }^{2,3}$ It does not, however, differentiate between arterial and venous obstruction. Pulmonary angiography is required to confirm pulmonary venous obstruction and is considered by some to be the investigation of choice when the diagnosis is suspected. ${ }^{3}$ Others have found transesophageal echocardiography to be useful in diagnosing pulmonary venous obstruction, demonstrating minimal flow in the affected vein and visualization of a thrombus. ${ }^{1,2}$ This investigation can be easily performed on the bedside and has the advantages of being quick and reliable, as well as not requiring any intravenous contrast.

The treatment options for pulmonary venous infarction after lung transplantation are often limited by the development of irreversible lung damage. Resection by lobectomy has been attempted after unilateral lung transplantation; however, the patient died 48 hours later. ${ }^{1}$ Retransplantation has been performed with success for the treatment of pulmonary venous infarction that involved the entire donor lung in a patient who had undergone unilateral lung transplantation. ${ }^{2}$ In our patient, the pulmonary venous infarction was treated conservatively because it involved only the apical segment of the right lower lobe, with the remainder of the donor lungs functioning adequately. The treatment of pulmonary venous infarction after lung transplantation needs to be tailored to the individual case and depends on the cause of the infarct, the extent of the infarct, and the remaining amount of unaffected donor lungs. If the cause of the infarct is correctable, such as a technical anastomotic problem, and the infarct involves the entire donor lung in a patient who has undergone unilateral lung transplantation, then the treatment should be surgical correction before irreversible damage, provided the diagnosis is made early, before established infarction has occurred. If irreversible damage has occurred, retransplantation is necessary. On the other hand, if the infarct has affected only a small part of the donor lung, such as in our case, with the remaining unilateral lung and the contralateral donor lung unaffected, then the treatment should be conservative, relying on sufficient amounts of unaffected lung and pulmonary reserve to get the patient through recovery from the lung transplantation. Thrombolytic therapy may be useful in treatment of a narrowing or occlusion of the anastomosis that has been caused by a thrombus in the absence of a surgically correctable problem.

In conclusion, we present a case of pulmonary segmentalvenous infarction after living-donor lung transplantation that was successfully treated with conservative means.

\section{References}

1. Sarsam MA, Yonan NA, Beton D, McMaster D, Deiraniya AK. Early pulmonary vein thrombosis after single lung transplantation. $J$ Heart Lung Transplant. 1993;12:17-9.

2. Malden ES, Kaiser LR, Gutierrez FR. Pulmonary vein obstruction following single lung transplantation. Chest. 1992;102:645-7.

3. Williamson WA, Tronic BS, Levitan N, Webb-Johnson DC, Shahian DM, Ellis FH Jr. Pulmonary venous infarction. Chest. 1992;102:937-40. 